\title{
BMJ Open Electroacupuncture to alleviate postoperative pain after a laparoscopic appendectomy: study protocol for a three-arm, randomised, controlled trial
}

\author{
Seunghoon Lee, ${ }^{1}$ Dongwoo Nam, ${ }^{1,2}$ Minsoo Kwon, ${ }^{1}$ Won Seo Park, ${ }^{3,4}$ \\ Sun Jin Park ${ }^{3,4}$
}

To cite: Lee S, Nam D, Kwon M, et al. Electroacupuncture to alleviate postoperative pain after a laparoscopic appendectomy: study protocol for a three-arm, randomised, controlled trial. BMJ Open 2017;7:e015286. doi:10.1136/ bmjopen-2016-015286

- Prepublication history for this paper is available online. To view these files, please visit the journal online (http://dx.doi. org/10.1136/bmjopen-2016015286).

Received 23 November 2016 Revised 13 June 2017 Accepted 13 June 2017

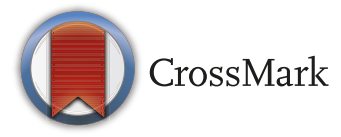

${ }^{1}$ Department of Acupuncture and Moxibustion Medicine, Kyung Hee University Korean Medicine Hospital, Seoul, South Korea

${ }^{2}$ Department of Acupuncture and Moxibustion, College of Korean

Medicine, Kyung Hee University,

Seoul, South Korea

${ }^{3}$ Department of Surgery, Kyung Hee University Hospital, Seoul, South Korea

${ }^{4}$ Department of Surgery, Kyung Hee University School of Medicine, Seoul, South Korea

Correspondence to

Professor Sun Jin Park;

gsdrpark@naver.com

\section{ABSTRACT}

Background The purpose of this study is to evaluate the efficacy and safety of electroacupuncture (EA) for postoperative pain after laparoscopic appendectomy compared with sham electroacupuncture (SEA) and no acupuncture treatment.

Methods and analysis This study is a protocol for a three-arm, randomised, patient-assessor-blinded (to the type of acupuncture treatment), controlled, parallel trial. 138 participants diagnosed with appendicitis and scheduled for laparoscopic appendectomy will be randomly assigned to the EA group $(n=46)$, SEA group $(n=46)$ or control group $(n=46)$. The EA group will receive acupuncture treatment at both regional and distal acupuncture points with electrostimulation. The SEA group will receive sham acupuncture treatment with mock electrostimulation. Both EA and SEA groups will receive a total of four treatments 1 hour preoperative, 1 hour postoperative and during the morning and afternoon the day after surgery with the same routine postoperative pain control. The control group will receive only routine postoperative pain control. The primary outcome is the 11-point Pain Intensity Numerical Rating Scale (PI-NRS) at 24 hours after surgery. The secondary outcomes are the PI-NRS, analgesic consumption, opioid-related side effects, time to first passing flatus, quality of life and adverse events evaluated 6, 12, 24 and 36 hours and 7 days after surgery.

Ethics and dissemination The study was planned in accordance with the Helsinki Declaration and the Korean Good Clinical Practice Guidelines to protect the participants and was approved by the institutional review board (IRB) of Kyung Hee University Medical Center (KMC IRB-1427-02). The results will be disseminated in peer-reviewed journals and presented at international conferences.

Trial registration number Clinical Research Information Service (KCT0001328).

\section{INTRODUCTION}

Laparoscopic surgery is a general surgical technique with advantages over traditional open surgery such as reduced postoperative pain, shorter hospital stay, faster recovery time, decreased postoperative ileus, reduced
Strengths and limitations of this study

- This study comprises real electroacupuncture with sham electroacupuncture using non-penetrating placebo needles to reduce performance bias.

- The electroacupuncture regimen was already used and validated in the clinic.

- Electroacupuncture treatment is difficult to blind practitioners.

scarring and preserved immune function. ${ }^{12}$ Among various factors, pain is the most important independent predictor of recovery time after laparoscopic surgery. ${ }^{3}$ Despite small incision size, laparoscopic surgery still results in substantial postoperative pain in the incision region (somatic pain). Moreover, inflation with carbon dioxide during the laparoscopic procedure induces shoulder pain due to irritation of the phrenic nerve, which is referred to as visceral pain. ${ }^{4}$

Multimodal approaches are beneficial for treating postoperative pain because there are multiple mechanisms of pain after laparoscopic surgery. ${ }^{3}$ Opioid analgesics are generally used to control pain with rapid onset of action. ${ }^{5}$ However, side effects associated with opioids such as nausea/vomiting, pruritus and reduction in bowel motility (leading to ileus and constipation) may lead to a delayed hospital discharge. ${ }^{6}$ Although other modalities, such as non-steroidal anti-inflammatory drugs, cyclooxygenase-2 inhibitors and local anaesthetics, are also used for postoperative pain, patients who have undergone laparoscopic surgery are generally unsatisfied with pain control and approximately $30 \%-40 \%$ patients suffer from moderate pain after hospital discharge. ${ }^{7}$

Electroacupuncture (EA) shows the potential to reduce postoperative pain as an adjuvant therapy to conventional 
anaesthetics. Several clinical trials have shown that preoperative or postoperative EA reduced postoperative pain and analgesic consumption with no significant adverse effects. ${ }^{8-11}$ However, based on a recent systematic review, there is still insufficient evidence to conclude that EA is an effective method for controlling postoperative pain in surgery due to methodological flaws in the studies that have been performed. Moreover, previous acupuncture trials have mostly focused on postoperative pain after open surgery ${ }^{12}$ or orthopaedic surgery such as total knee arthroplasty ${ }^{13-15}$ or back surgery. ${ }^{16}$ For this reason, despite the potential benefits of EA for postoperative pain, the efficacy and safety of EA for laparoscopic surgery remains to be examined. We aim to evaluate the effects of EA on postoperative pain after laparoscopic appendectomy as a basic, common and representative laparoscopic surgery.

\section{METHODS/DESIGN \\ Objective}

The aim of this study is to assess the efficacy and safety of EA for postoperative pain through pain intensity, analgesic consumption, opioid-related side effects, time to first passing flatus, quality of life and adverse events after laparoscopic appendectomy compared with sham electroacupuncture (SEA) and no acupuncture treatment.

\section{Design and setting}

This study is a single-centre, three-arm, equal randomised, patient-blinded and assessor-blinded (to the type of acupuncture treatment), parallel-group, clinical trial conducted in Korea.

\section{Recruitment period}

The participants will be recruited from the Kyung Hee University Medical Center in Seoul, Korea. Recruitment is expected to span from April 2015 to March 2017.

\section{Methods of recruitment}

A total of 138 participants diagnosed with appendicitis and scheduled for laparoscopic appendectomy through the outpatient department or emergency room will be recruited at the Department of Surgery of Kyung Hee University Medical Center.

\section{Study setting}

Oral and written consent will be obtained from patients who are potentially eligible for the study before surgery. A researcher will explain the two types of EA treatments as 'classical EA, typically used in Korean medicine clinics' and 'non-classical EA, rarely used in Korean medicine clinics'. ${ }^{17}$ After a patient voluntarily consents to the study, a researcher will screen whether the patient can participate in the study. If the patient satisfies the inclusion/exclusion criteria, a clinical research coordinator (CRC) will contact an independent researcher who has the random number table, and the patient will be randomly allocated into one of three groups (the EA, SEA or control group) at a 1:1:1 allocation ratio. Only the patients assigned to the EA or SEA group will receive the acupuncture treatment 1 hour before the scheduled laparoscopic surgery. The postoperative pain management of all patients will be performed according to the manual of the Department of Surgery of the Kyung Hee University Medical Center. The patients assigned to the EA or SEA groups will receive acupuncture treatments again 1 hour after surgery and the morning and afternoon of the following day. Patients will be discharged on 2 days after surgery, and follow-up visits will be scheduled on 7 $( \pm 3$ ) days after surgery (figure 1 ).

\section{Participants}

\section{Inclusion criteria}

Participants who meet the following conditions will be included: (1) men or women aged 19 to 65 years, (2) diagnosed with appendicitis and scheduled for laparoscopic appendectomy and (3) agreement via written informed consent after being provided with an explanation regarding the purpose and characteristics of this study.

\section{Exclusion criteria}

Participants who have experienced or have one or more of the following conditions will be excluded: (1) accompanying complications such as perforated appendicitis or diffuse peritonitis, (2) development of a surgical site infection during the hospital stay, (3) regular use of painkillers or use of other pain relieving medicine on the day of the surgery, (4) cardiovascular disorders such as arrhythmia or use of a pacemaker, (5) received acupuncture treatment within 6 weeks prior to surgery, (6) known hypersensitive reaction to acupuncture treatment or the inability to cooperate with the acupuncture procedure, (7) pregnant, breast feeding or expecting a pregnancy during the study period and (8) others that have been deemed inadequate for participation by research investigators.

\section{Randomisation and allocation concealment}

Enrolled participants will be randomly assigned to EA, SEA or control group (1:1:1). An independent, blinded statistician will generate the block randomisation scheme.

The table will be managed by another independent researcher who is not involved in the recruitment, acupuncture treatment or assessment. The CRC will send the assignment information to the researcher who will conduct random allocation, then the researcher will only provide assignment information to the doctors of Korean medicine (DKMs) that perform the acupuncture treatment. To ensure allocation concealment, the allocated group information will be recorded in an allocation $\log$ by the researcher and will not be opened until the data are locked.

\section{Blinding}

The participants will be blinded to the type of acupuncture treatment and the assessor, data managers, statisticians and study monitors will be blinded to the allocation. All participants who receive acupuncture will be treated using the Park sham device (PSD; Acuprime, 


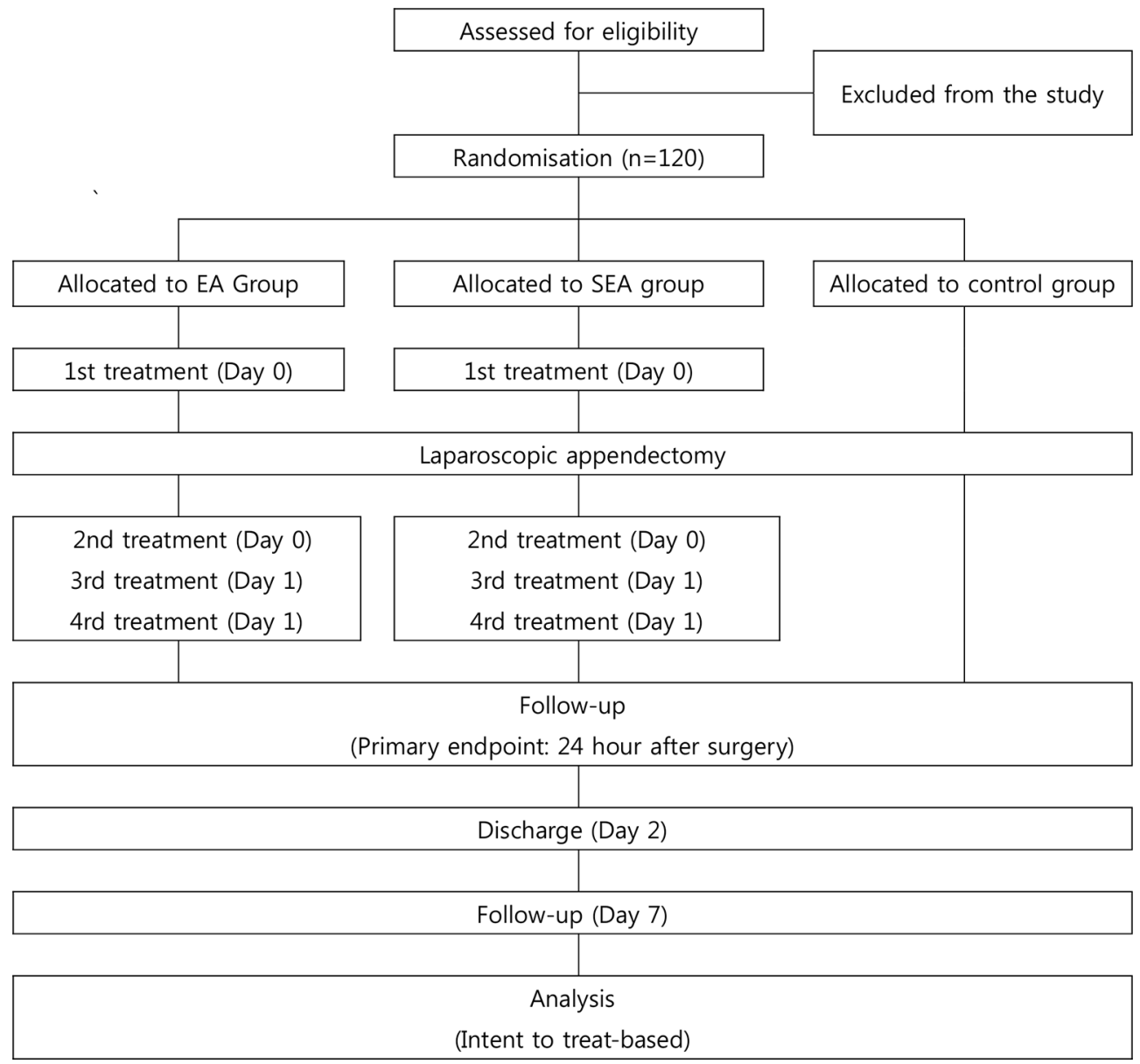

Figure 1 Flow chart of study process. EA, electroacupuncture; SEA, sham electroacupuncture.

Exeter, UK) regardless of what group they are included in. Moreover, mock EA will be provided with the same pulse sound and lamp light as real EA, so the participants will not be able to predict the allocated group based on the appearance of the acupuncture treatment. The blinding will be maintained until the data are locked. For blinding evaluation, allocation guessing will be assessed immediately after the final treatment. Practitioners and assessors will be instructed to treat the participants according to predefined standard operating procedures (SOPs) during the trial to maintain blinding.

\section{Intervention}

EA treatment will be conducted by DKMs with more than 6 years of Korean medicine college education and at least 2 years of clinical experience. A total of four acupuncture treatments will be performed in the EA and SEA groups. One hour preoperative and 1 hour postoperative treatments will be performed on the day of the surgery (day 0 ). On the day after surgery (day 1 ), one treatment will be carried out in the morning (08:00-12:00) and another in the afternoon (13:00-17:00) with at least 4 hours between treatments. Each acupuncture treatment will be performed after the acupuncture points are sterilised with a disposable ethyl alcohol swab and the acupuncture needles will be retained for $30 \mathrm{~min}$. Both EA and SEA are performed under the same conditions, except for the needling components, ${ }^{18}$ and both groups will be given the same postoperative analgesics in accordance with the predefined protocol and SOPs. This information will be given to the practitioners during workshops before the study begins to ensure standardisation of the treatments.

A Park sham needle guide tube ${ }^{1920}$ will be used for both EA and SEA groups to ensure that the patients remain unaware of the differences between the two acupuncture treatments. This will ensure that the patients do not discern any differences between the individualised, real acupuncture and sham acupuncture treatments, although the penetration of the needles will vary between the two groups.

\section{Surgical procedure}

The surgical department is part of a tertiary teaching hospital, in which 12 surgeons and 7 residents participate. Laparoscopic appendectomies are performed by surgeons or residents under the supervision of a surgeon. The operation is performed under general anaesthesia with the patient in a supine position. A $12 \mathrm{~mm}$ trocar for the camera is inserted just below the umbilicus. Two additional $5 \mathrm{~mm}$ trocars are inserted in the suprapubic area and right or left lower abdomen. The surgical procedure is performed using standard laparoscopic instruments and in a usual manner including dividing the mesoappendix, ligating the appendix and removing the specimen using 


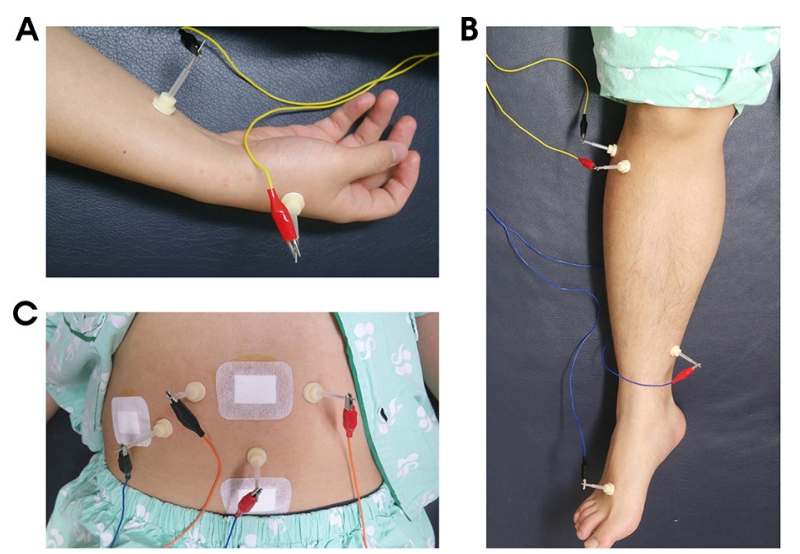

Figure 2 Acupuncture points of treatment. (A) The hands will be treated at two standard acupuncture points (LI4 and PC6). (B) The legs will be treated at four standard acupuncture points (ST36, GB34, SP6 and LR3). (C) The abdomen will be treated four ashi points located within a diameter of $5 \mathrm{~cm}$ from the incision site. (A written consent for the picture was obtained from the pictured subject. Photograph by Seunghoon Lee).

a pouch. The $12 \mathrm{~mm}$ port site is closed using 2-0 Vicryl sutures and the skin incision is closed with subcuticular sutures.

\section{Electroacupuncture treatment}

A total of four acupuncture sessions will be performed using $0.25 \times 40 \mathrm{~mm}$ disposable sterile acupuncture needles (Dong Bang Acupuncture, Chungnam, South Korea). After penetration, deqi sensation will be induced using reinforcing-reducing techniques such as rotating and lifting-thrusting methods. An EA device (Partner-1; ITC, Daejeon, South Korea) will be used to apply $2 / 120 \mathrm{~Hz}$ (acupuncture points: bilateral ST36, GB34, LI4, PC6, SP6, LR3) and $120 \mathrm{~Hz}$ (acupuncture points: four ashi points located within a diameter of $5 \mathrm{~cm}$ from the incision site) at $80 \%$ of the maximum intensity that the patient can endure. Acupuncture point selection (including local abdomen and distal points) and frequency of electrical stimulation were based on traditional acupuncture theory and clinical experience by clinicians. The practitioner will regulate the intensity based on the degree of the twitching of muscles or by request from the subject, and only one adjustment will be allowed during treatment. In both acupuncture groups, an infrared lamp will be applied to keep the abdominal area of the patients warm during treatment (figure 2).

\section{Sham electroacupuncture treatment}

PSD will be used for SEA treatment. It is a validated sham acupuncture device that consists of two tubes and a sham acupuncture needle. ${ }^{20}$ The 'Guide tube' supports the needle as it penetrates the skin vertically. A larger tube called the 'Park tube' is attached to the ring base and allows the 'Guide tube' to move along the 'Park tube.' A silicon base is attached to the skin using double-sided tape. ${ }^{19}$ A sham acupuncture needle of PSD is indistinguishable from a real acupuncture needle, but it does not penetrate the skin.

For local abdomen points, SEA treatment will be performed on the abdomen region (two sites; $3 \mathrm{~cm}$ above and $2 \mathrm{~cm}$ lateral to the umbilicus). For distal points, both arms (two sites per arm; $5 \mathrm{~cm}$ and $7 \mathrm{~cm}$ below the midpoint of the cubital crease) and both legs (two sites; $7 \mathrm{~cm}$ above the medial malleolus and $0.3 \mathrm{~cm}$ lateral to the tibia and $9 \mathrm{~cm}$ above the medial malleolus and $0.3 \mathrm{~cm}$ lateral to the tibia) will be treated using the PSD and sham acupuncture needles creating a false movement that is similar to the twisting manoeuvre of acupuncture. No deqi sensation will be induced. To simulate electrical stimulation, an electrical stimulating device will be connected to the needles, but no electrical current will be delivered. The patient will see the light and hear the sound of the pulse generator equal to those of the EA group. The rest of the procedure is equal to that of EA group.

\section{Postoperative pain control}

All participants will receive standard postoperative pain control in accordance with the manual of the Department of Surgery at Kyung Hee University Medical Center. The standard postoperative pain control procedure is as follows.

Nefopam hydrochloride (ACUPAN INJ $20 \mathrm{mg} / 2 \mathrm{~mL}$; Pharmbio Korea, Chungju, South Korea) and Ketorolac tromethamine (KETOCIN INJ $30 \mathrm{mg} / \mathrm{mL}$; Myungmoon Pharm., Seoul, South Korea) will be routinely used to control postoperative pain. Nefopam hydrochloride $100 \mathrm{mg}$ and Ketorolac tromethamine $60 \mathrm{mg}$ will be mixed and diluted in $500 \mathrm{~mL}$ dextrose and given intravenously twice during the hospitalisation period.

If additional pain control is needed, tramadol hydrochloride (TRAMADOL HCL INJ $50 \mathrm{mg} / \mathrm{L}$; Shin Poong Pharm, Seoul, South Korea) $50 \mathrm{mg}$ (intramuscular or intravenous injection) will be administered no more than once every $4-5$ hours, and the maximum dose of tramadol $\mathrm{HCl}$ will not exceed $400 \mathrm{mg}$ per day. The total amount of tramadol use will be recorded by a blinded researcher.

\section{Outcome measures}

The details of the outcome measures and time points are shown in table 1 .

\section{Primary outcome}

The primary outcome is pain intensity in the abdomen region using the 11-point Pain Intensity Numerical Rating Scale (PI-NRS; $0=$ no pain and $10=$ worst possible pain, 11-point Likert scale) 24 hours after surgery. The PI-NRS has been widely used to assess all kinds of pain, and recently it has been validated for measuring postoperative pain intensity. ${ }^{21}$ The patients are asked to choose a value that best represents the intensity of pain that they are experiencing at the moment. A written form with numeric values from 0 to 10 is frequently used as well.

In a previous study, a threshold of NRS $\geq 4$ was the cut-off value for distinguishing mild and moderate-to-severe postoperative pain intensity during the first 24 hours after 


\begin{tabular}{|c|c|c|c|c|c|}
\hline Period & $\mathbf{S}$ & & $\mathbf{T}$ & & $\mathbf{F}$ \\
\hline Day & 0 & 0 & 1 & 2 & 7 \\
\hline Informed consent & ○ & & & & \\
\hline Demographic characteristics & ○ & & & & \\
\hline Inclusion/exclusion criteria & 0 & & & & \\
\hline Conformity assessment & 0 & & & & \\
\hline Random allocation & & 0 & & & \\
\hline Acupuncture treatment & & ० & $\circ$ & & \\
\hline Laparoscopic surgery & & 0 & & & \\
\hline \multicolumn{6}{|l|}{ Efficacy assessments } \\
\hline PI-NRS & & 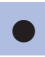 & 0 & 0 & 0 \\
\hline Consumption of analgesics & & ○ & ○ & ○ & \\
\hline Opioid-related side effects & & ○ & ○ & 0 & \\
\hline Time to first passing flatus & & $\bullet$ & 0 & ○ & \\
\hline EQ-5D & & 0 & 0 & ? & 0 \\
\hline Blinding test & & & & ० & \\
\hline Safety assessment & & 0 & 0 & 0 & 0 \\
\hline
\end{tabular}

Day 0: surgery day; day 1: 1 day after surgery; day 2: 2 days after surgery; day $7: 7$ days after surgery ( \pm 3 days).

Acupuncture treatment will be conducted two times at day

1. Efficacy assessments will be conducted 6, 12, 24, 36 and

72 hours after surgery during the treatment period.

- All groups.

- Both the electroacupuncture and sham acupuncture groups. EQ-5D, EuroQol Five Dimensions Questionnaire; F, follow-up visit; PI-NRS, 11-point Pain Intensity Numerical Rating Scale; S, screening visit; $\mathrm{T}$, treatment period.

surgery. This value was confirmed using four different methodological approaches. ${ }^{22}$

In cases where additional pain medicine is administered, the assessors will be instructed to evaluate pain intensity 2 hours after drug administration.

\section{Secondary outcomes}

The pain intensity of the abdomen region at rest, coughing and overall average will be measured by the PI-NRS at 6 , 12 , and 36 hours and 7 days after surgery. Moreover, laparoscopy-induced shoulder pain will also be measured 6 , 12, 24, 36 hours and 7 days after surgery. We predefined the type of improvement for clinical relevance as follows: a $30 \%$ reduction in PI-NRS is 'minimal improvement'; a $70 \%$ reduction in PI-NRS is 'much improvement' and a $90 \%$ reduction in PI-NRS is 'complete improvement."23 24 Therefore, in this trial, both the absolute and relative score changes will be used to analyse the clinical relevance.

The total amount of analgesic consumption used will be evaluated by an independent researcher blinded to the allocation at $6,12,24$ and 36 hours and 7 days after surgery.
The opioid-related side effects such as nausea, vomiting, itching and ileus will be measured by PI-NRS at 6, 12, 24, 36 hours and 7 days after surgery.

Time-to-first-passing flutus first after surgery will be checked.

The EuroQol Five Dimensions Questionnaire (EQ-5D) will be used to evaluate the quality of life of a patient with postoperative pain at 24 hours and 7 days after surgery. EQ-5D is a standardised tool used to measure health outcomes that includes generic questions about quality of life as it relates to personal health status. EQ-5D is regarded as one of the most appropriate instruments to evaluate patient quality of life after surgery. ${ }^{25}$ We will use the Korean version of the EQ-5D. ${ }^{26}$

\section{Safety and adverse events}

The practitioners will be instructed to record all unexpected and unintended responses that are not necessarily related to the EA treatment on an adverse event report form. Pain, bruising, bleeding, dizziness, anxiety and infection are some of the adverse events known to be related to EA treatments. ${ }^{27} \mathrm{~A}$ causal relationship between the EA treatment and adverse events will be assessed using a 6 -point scale (1=definitely related, $2=$ probably related, $3=$ possibly related, $4=$ probably not related, $5=$ definitely not related and $6=$ unknown), and the severity of the adverse events will be scored using a 4-point scale ( $1=$ mild, $2=$ moderate, $3=$ severe and $4=$ extremely severe).

\section{Blinding assessment}

At the end of treatment, the patients will guess to which group they were allocated: real or sham EA treatment. The patients will choose one of the following three options based on their personal feelings about the treatment they received: 'classical acupuncture typically used in Korean medicine clinics', 'non-classical acupuncture, rarely used in Korean medicine clinics' or 'do not know'.

\section{Sample size calculation}

The sample size was calculated based on the mean and SD of NRS from the previous study. ${ }^{9}$ The mean and SD of the EA group (2.1 and 1.2) and those of the control group (3.2 and 1.8) were used as the expected values in our study. With a two-sided significance level of $5 \%(\alpha=0.05)$ and $80 \%$ power $(1-\beta=0.8), 32$ patients are required per treatment group. Considering a $30 \%$ dropout rate, a total of 138 patients are needed for the study. The software PASS V.12 (NCSS, Kaysville, Utah, USA) was used for the calculation.

\section{Statistical analysis \\ Analysis populations}

The analysis set will include a full-analysis set (FAS), per-protocol (PP) set and safety set. The safety set will consist of all randomised participants who received laparoscopic surgery or at least one EA, SEA or no acupuncture (NA) treatment during the course of the study. The FAS population will consist of all participants in the safety population who are evaluable for the primary outcome. The FAS population will be used as the 
primary population for all efficacy analyses. The PP population will consist of the all participants included in the FAS population, but will exclude the following: (1) participants violating any inclusion/exclusion criterion and (2) participants with major protocol violations (eg, poor compliance $(<75 \%$ treatment compliance), incorrect completion of study); only sufficiently serious violations will warrant exclusion.

\section{General statistical methodology}

Descriptive summaries will be provided where appropriate for each of the primary and secondary outcomes. In general, summaries will be presented by the participant population and by treatment groups and/or overall. In general, continuous variable summaries will include the number of participant, mean, SD, median, minimum and maximum and first and third quartiles, as appropriate. Categorical variable summaries will include the frequency and percentage of participants who are in the particular category.

The last observation carried forward method will be used to process the missing data for the primary outcome. All hypothesis testing will be carried out at the $5 \%$ (two-sided) significance level. All secondary outcomes are exploratory and therefore no adjustment for multiple testing will be applied.

\section{Statistical software}

Data manipulation, statistical summaries and statistical analyses will be performed using SAS V.9.4 by an independent biostatistician. Some analysis may be carried out in R V.3.2.0 or higher (https:/ /www.r-project.org/).

\section{Analysis of demographics and other baseline characteristics}

Comparisons of demographic and other baseline characteristics among the three groups will be made using the $\chi^{2}$ test, Fisher's exact test, analysis of variance or Kruskal-Wallis test, according to the type of variable.

\section{Primary efficacy outcome analysis}

For a confirmatory analysis, an a priori-ordered two-sided null hypothesis will be tested using the Student's t-test or Wilcoxon rank sum test in a stepwise fashion with a significance level of $5 \% .{ }^{928}$ First, whether EA was more efficacious than no acupuncture treatment in reducing postoperative pain 24 hours after laparoscopic surgery will be investigated and second (only if the first null hypothesis was rejected), whether EA was more efficacious than SA will be investigated. All efficacy analyses will use the FAS and the PP population.

\section{Secondary outcome analysis}

The secondary outcomes will be evaluated using a Student's t-test or Wilcoxon rank sum test for continuous data or $\chi^{2}$ test or Fisher's exact test for categorical data. These results will be compared with an adjusted result using an analysis of covariance with the baseline measurements (eg, PI-NRS before surgery, type of appendicitis (suppurative, exudative, gangrenous or perforated) and age) as a covariate, the treatment group as a fixed effect. Multivariate analysis using a mixed model for repeated measures will be performed for repeated measure outcomes.

\section{Data and safety monitoring}

To ensure the quality of the data is in accordance with the predetermined protocol and SOPs, regular monitoring will be carried out. Monitors will be blinded to the allocation and will examine whether the recruitment procedures and data recording followed the protocol in the case report forms. In case modifications in the study methods are necessary, such as changes to the eligibility criteria, treatment regimens or duration of follow-up, the principal investigator may discuss the issue with independent researchers and statisticians. In case of severe adverse events or crucial issues, the principal investigator will determine whether the events are acceptable or whether it is necessary to change or terminate the trial.

\section{Ethics and dissemination}

The study was planned in accordance with the Helsinki Declaration and the Korean Good Clinical Practice Guidelines to protect the participants and was approved by the institutional review board (IRB) of Kyung Hee University Medical Center (KMC IRB-1427-02). The participants will be informed on the potential benefits, risks, alternatives and responsibilities of the study by the researchers during the consent process. To avoid potential adverse events, if there is a patient whom the practitioner considers unsuitable for EA treatment due to an abnormal health condition such as severe pain or vomiting, the treatment will be rescheduled within 2 hours at the practitioner's discretion. The findings will be disseminated in peer-reviewed journals and conference presentations.

\section{DISCUSSION}

The purpose of this study is to evaluate the efficacy and safety of EA for postoperative pain after laparoscopic appendectomy compared with SEA and no acupuncture treatment. When planning an EA study, timing of treatment, treatment points and frequency of electric stimulation should all be carefully considered as these can affect the outcomes of EA. In several EA trials for postoperative pain, it is likely that the results varied because of heterogeneity in the protocol. For this reason, we established a regimented EA protocol based on previous research and our clinical experience. This protocol has been tested and optimised at the Kyung Hee University Medical Center.

While acupuncture is usually applied after the onset of pain in most pain-related conditions such as low back pain, knee pain or headache, acupuncture can be performed before, during or after surgery to alleviate postoperative pain. Most preoperative and postoperative acupuncture trials have shown to be effective for reducing postoperative pain and analgesic consumption. However, intraoperative EA has shown little to no effect on analgesic consumption, though only a few studies have been conducted. Adding the fact that it is realistically difficult 
to conduct EA during surgery, we planned to perform EA treatments only before and after surgery.

Previous studies have used various acupuncture points that include distal points such as ST36 and LI $4,{ }^{8911} 29$ local points around the incision region ${ }^{29}$ or a combination of points. ${ }^{10} 30$ There have not yet been any studies that directly compare the effects of distal and local points, but one study ${ }^{29}$ reported that electrical nerve stimulation significantly reduced postoperative pain in both local and distal point treatment groups. Some studies used only distal points due to fear of potential adverse events by direct electrical stimulation around the incision site, but there has not been any reports of adverse events such as increased pain or infection of the surgical site in studies that have applied electrical stimulation directly near the surgical region. ${ }^{10}{ }^{29}$ Therefore, we utilised both local incision points as well as distal acupuncture points to maximise the effect of acupuncture treatment.

There is still a lot of debate on the optimal frequency of EA for pain control. Individual studies have used low, high or mixed frequencies and the effectiveness varies from study to study. In the case of EA at distal acupuncture points, the low frequency of $2 \mathrm{~Hz}$ generally releases $\beta$-endorphins, and high frequency (over $100 \mathrm{~Hz}$ ) is known to release dynorphins. Therefore, a mixed combination of both low and high frequencies releases various opioid peptides and creates a synergetic effect that inhibits pain. ${ }^{27} 31$ On the other hand, in case of EA around the incision site, high-frequency electrical stimulation (over $100 \mathrm{~Hz}$ ) stimulates specific afferent nerve fibres instead of releasing endogenous opioid peptide and has been shown to be effective for superficial parietal pain caused by skin incision. ${ }^{31}$ For this reason, one study suggested that mixed stimulation of local high frequency and remote low frequency by silver spike point offered the most relief for postoperative pain. ${ }^{32}$ As a result, we are using different frequencies from two separate EA devices for local and distal points to maximise the effect of EA.

We expect that if the results show that EA treatment is a safe and effective option for reducing postoperative pain and analgesic consumption, the study may act as evidence to support the inclusion of EA treatment in a future 'enhanced recovery after surgery' programme in conjunction with laparoscopic appendectomy.

\section{Acknowledgements The authors thank Professor Kun Hyung Kim for encouraging suggestions and helpful comments, and Hyun Jin Kim, PA, of Department of Surgery of Kyung Hee University Hospital for her assistance with the data collection.}

Contributors SL and SJP planned the overall study protocol. SL drafted the manuscript. SL, DN, MK, WSP and SJP participated in critical revision of the manuscript. SJP had the final responsibility for the decision to submit for publication. All of the authors have read and approved the final manuscript.

Funding This work was supported by a grant from Kyung Hee University in 2014 (KHU-20140706).

Competing interests None declared.

Patient consent Obtained.

Ethics approval The institutional review board (IRB) of Kyung Hee University Medical Center (KMC IRB-1427-02).
Provenance and peer review Not commissioned; externally peer reviewed.

Data sharing statement We will share the data after the trial is finished. The full data set will be available by an author contact when this trial is completed and published.

Open Access This is an Open Access article distributed in accordance with the Creative Commons Attribution Non Commercial (CC BY-NC 4.0) license, which permits others to distribute, remix, adapt, build upon this work non-commercially, and license their derivative works on different terms, provided the original work is properly cited and the use is non-commercial. See: http://creativecommons.org/ licenses/by-nc/4.0/

(C) Article author(s) (or their employer(s) unless otherwise stated in the text of the article) 2017. All rights reserved. No commercial use is permitted unless otherwise expressly granted.

\section{REFERENCES}

1. Yoo J. Laparoscopic colorectal surgery. Perm J 2008;12:27-31.

2. Li L, Tian J, Tian H, et al. The efficacy and safety of different kinds of laparoscopic cholecystectomy: a network meta analysis of 43 randomized controlled trials. PLoS One 2014;9:e90313.

3. Mitra S, Khandelwal P, Roberts K, et al. Pain relief in laparoscopic cholecystectomy--a review of the current options. Pain Pract 2012;12:485-96.

4. Tsai HW, Chen YJ, Ho CM, et al. Maneuvers to decrease laparoscopy-induced shoulder and upper abdominal pain: a randomized controlled study. Arch Surg 2011;146:1360-6.

5. Hutchison R, Chon EH, Tucker, Jr W, et al. A comparison of a fentanyl, morphine, and hydromorphone patient-controlled intravenous delivery for acute postoperative analgesia: a multicenter study of opioid-induced adverse reactions. Hosp Pharm 2006;41:659-63.

6. Garimella V, Cellini C. Postoperative pain control. Clin Colon Rectal Surg 2013;26:191-6.

7. Rawal N, Allvin R, Amilon A, et al. Postoperative analgesia at home after ambulatory hand surgery: a controlled comparison of tramadol, metamizol, and paracetamol. Anesth Analg 2001;92:347-51.

8. Lin JG, Lo MW, Wen YR, et al. The effect of high and low frequency electroacupuncture in pain after lower abdominal surgery. Pain 2002;99:509-14.

9. Ng SS, Leung WW, Mak TW, et al. Electroacupuncture reduces duration of postoperative ileus after laparoscopic surgery for colorectal cancer. Gastroenterology 2013;144:307-13.

10. Sim CK, Xu PC, Pua HL, et al. Effects of electroacupuncture on intraoperative and postoperative analgesic requirement. Acupunct Med 2002;20(2-3):56-65.

11. Wong RH, Lee TW, Sihoe AD, et al. Analgesic effect of electroacupuncture in postthoracotomy pain: a prospective randomized trial. Ann Thorac Surg 2006;81:2031-6.

12. Sun Y, Gan TJ, Dubose JW, et al. Acupuncture and related techniques for postoperative pain: a systematic review of randomized controlled trials. Br J Anaesth 2008;101:151-60.

13. Chen CC, Yang CC, Hu CC, et al. Acupuncture for pain relief after total knee arthroplasty: a randomized controlled trial. Reg Anesth Pain Med 2015;40:31-6.

14. Liu GP, Xue FS, Sun C, et al. Electroacupuncture for pain treatment after total knee arthroplasty. Acupunct Med 2015;33:433.

15. Tzeng CY, Chang SL, Wu CC, et al. Single-blinded, randomised preliminary study evaluating the effects of $2 \mathrm{~Hz}$ electroacupuncture for postoperative pain in patients with total knee arthroplasty. Acupunct Med 2015;33:284-8.

16. Cho $\mathrm{YH}, \mathrm{Kim} \mathrm{CK}, \mathrm{Heo} \mathrm{KH}$, et al. Acupuncture for acute postoperative pain after back surgery: a systematic review and meta-analysis of randomized controlled trials. Pain Pract 2015;15:279-91.

17. Shen J, Wenger N, Glaspy J, et al. Electroacupuncture for control of myeloablative chemotherapy-induced emesis: A randomized controlled trial. JAMA 2000;284:2755-61.

18. Langevin HM, Wayne PM, Macpherson $\mathrm{H}$, et al. Paradoxes in acupuncture research: strategies for moving forward. Evid Based Complement Alternat Med 2011;2011:1-11.

19. Zhu D, Gao Y, Chang J, et al. Placebo acupuncture devices: considerations for acupuncture research. Evid Based Complement Alternat Med 2013;2013:1-9.

20. Park J, White A, Stevinson C, et al. Validating a new non-penetrating sham acupuncture device: two randomised controlled trials. Acupunct Med 2002;20:168-74.

21. Breivik $\mathrm{H}$, Borchgrevink $\mathrm{PC}$, Allen $\mathrm{SM}$, et al. Assessment of pain. $\mathrm{Br} \mathrm{J}$ Anaesth 2008;101:17-24. 
22. Gerbershagen HJ, Rothaug J, Kalkman CJ, et al. Determination of moderate-to-severe postoperative pain on the numeric rating scale: a cut-off point analysis applying four different methods. $\mathrm{Br} J$ Anaesth 2011:107:619-26.

23. Sloman R, Wruble AW, Rosen G, et al. Determination of clinically meaningful levels of pain reduction in patients experiencing acute postoperative pain. Pain Manag Nurs 2006;7:153-8.

24. Dworkin RH, Turk DC, Wyrwich KW, et al. Interpreting the clinical importance of treatment outcomes in chronic pain clinical trials: immpact recommendations. J Pain 2008;9:105-21.

25. Taylor RS, Ullrich K, Regan S, et al. The impact of early postoperative pain on health-related quality of life. Pain Pract 2013;13:515-23.

26. Kim MH, Cho YS, Unm WS, et al. Cross-cultural adaptation and validation of the Korean version of the EQ-5D in patients with rheumatic diseases. Qual Life Res 2005;14:1401-6.
27. White A, Cummings TM, Filshie J. An introduction to Western Medical Acupuncture. Churchill Livingstone/Elsevier,, 2008.

28. Brinkhaus $\mathrm{B}$, Witt CM, Jena $\mathrm{S}$, et al. Acupuncture in patients with chronic low back pain: a randomized controlled trial. Arch Intern Med 2006;166:450-7.

29. Chen L, Tang J, White PF, et al. The effect of location of transcutaneous electrical nerve stimulation on postoperative opioid analgesic requirement: acupoint versus nonacupoint stimulation. Anesth Analg 1998;87:1129-34.

30. Wang B, Tang J, White PF, et al. Effect of the intensity of transcutaneous acupoint electrical stimulation on the postoperative analgesic requirement. Anesth Analg 1997;85:406-13.

31. Mayor DF. Electroacupuncture: a Practical Manual and Resource. Elsevier Churchill Livingstone, 2007.

32. Taguchi R. Acupuncture anesthesia and analgesia for clinical acute pain in Japan. Evid Based Complement Alternat Med 2008;5:153-8. 